\title{
Effect of abrasive particle bluntness on nano finishing using abrasive flow finishing (AFF) process
}

\author{
Deepu Kumar, Sachin Singh and M. Ravi Sankar* \\ Department of Mechanical Engineering, \\ Indian Institute of Technology Guwahati, 781 039, India \\ *Email: evmrs@iitg.ernet.in
}

\begin{abstract}
Abrasive flow finishing (AFF) process is an advanced fine finishing process, which finishes the simple to complex features using polymer rheological abrasive medium (medium). Medium consists of base polymers, plasticizers, softeners, hardeners, and abrasive particles. The medium should possess three basic properties i.e., flow ability, self deformability, and better abrading ability to finish in nano scale. The finishing action in AFF process depends on input parameters (extrusion pressure and number of cycles). These input parameters enhance the medium properties (flow and abrading abilities) from their base level. But abrading also depends on the sharpness of abrasive particle's cutting edge. As the extrusion pressure and number of cycles increases, the abrasive particle cutting edge sharpness reduces i.e., bluntness increases. The finishing rate gradually decreases with increase in abrasive particle cutting edge bluntness.
\end{abstract}

So in the present paper, comparative study among experimental, simulated with and without abrasive particle cutting edge bluntness is studied. Experimental finishing studies are carried out by varying extrusion pressure, number of strokes and plasticizer weight percentage in the medium. Later the medium is collected and abrasive particle cutting edge bluntness is experimentally determined using optical microscope at various cutting edges of abrasive particle to calculate average value of bluntness. The abrasive particle cutting edge bluntness increases with extrusion pressure and number of AFF strokes.

Later FE modeling and simulation is carried out with and without considering the abrasive particle cutting edge bluntness. Then this simulation results are compared with the experimental results (Figure 1). The average error percentage between experimental and simulation considering the abrasive particle bluntness is $2.90 \%$. But without considering the abrasive particle bluntness the error is $7.40 \%$. As the number of strokes increases, the finishing rate gradually decreases (after few strokes), this phenomenon is well agreement with simulation results considering the abrasive particle bluntness but not in agreement with simulation results without considering abrasive particle bluntness.

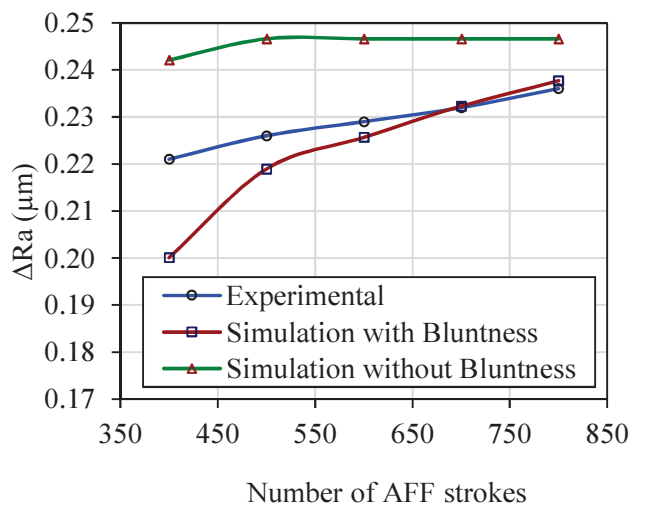

Figure 1: Effect of number of AFF strokes on change in surface roughness $(\Delta \mathrm{Ra})$

Acknowledgements: The authors are thankful for the financial support provided by Indian Institute of Technology Guwahati (SG/ME/P/MRS/01), Board of Research in Nuclear Sciences (ME/P/MRS/02), Department of Electronics \& Information Technology (Centre for Excellence in Research and Development of Nanoelectronic Theranostic Devices), and Department of Science and Technology for "Technology Systems Development Programme (DST/TSG/AMT/2015/619)". 